\title{
El soft law y su aplicación en el derecho comercial internacional ${ }^{1}$
}

\section{Soft law and its application in commercial international law}

\section{Bárbara Andrea Cortés Cabrera}

barbara.cortes.cabrera@gmail.com

Licenciada en Derecho con mención en Derecho Regulatorio. Minor en Gestión de Empresas. Universidad Alberto Hurtado.

Resumen: Dentro del agitado ritmo de profundas transformaciones que se encuentra experimentando el derecho internacional, el soft law emerge como una alternativa al más tradicional hard law. Poco a poco el soft law ha ido adquiriendo un mayor protagonismo en el escenario jurídico internacional, posicionándose como una herramienta de la que gozan las partes para conciliar acuerdos revestidos de voluntariedad pero no de vinculatoriedad. De este modo, el soft law se compone de un amplio espectro de instrumentos, los que encuentran aceptación en los diferentes sistemas jurídicos, en variadas áreas del derecho y dan respuesta transversalmente a las necesidades de los diferentes interesados. Por lo anterior, resulta relevante analizar esta nueva corriente a nivel internacional y su incorporación dinámica en instrumentos jurídicos.

Palabra clave: derecho internacional, soft law, derecho comercial internacional.

Abstract: Within the agitated rhythm of profound transformations that international law is experiencing, soft law emerges as an alternative to the more traditional hard law. Little by little, soft law has been acquiring a greater role in the international law scene, positioning itself as a tool that parties count with to generate agreements that are voluntary, but not binding. In this way, soft law is comprised by a large spectrum of instruments, which are accepted in varied legal systems, in several areas of law and give a transversal response to the different needs of stakeholders. For the aforementioned, it becomes relevant to analyze this new current on an international level and its dynamic inclusion in legal instruments.

Keywords: international law, soft law, international commercial law.

\footnotetext{
Artículo recibido el 15.03.2014 y aceptado el 17.10.2014.
} 
Los hombres, pudiendo ser desiguales en fuerza o en talento, se hacen iguales por convención y por derecho.

Jean-Jacques Rousseau

\section{Aspectos preliminares}

Una de las áreas jurídicas más influenciadas por el fenómeno de la globalización y por el nuevo orden mundial ha sido el derecho internacional, permeado por diferentes transformaciones como la revolución tecnológica, la economía abierta, el multilateralismo, etc.

Así, uno de los aspectos en que lo anterior se manifiesta con mayor intensidad es en el clásico papel de la ley como instrumento efectivo de conformación imperativa de conductas, instituciones y políticas sociales ${ }^{2}$.

Consecuentemente, estas transformaciones también han provocado cambios en los sujetos, fuentes y responsabilidad del derecho internacional, cambiando asimismo el objeto de su estudio ${ }^{3}$.

La omnipresente intervención del Estado queda hoy en entredicho debido a la creciente complejidad de la sociedad y a la consiguiente dificultad para que la intervención normativa resuelva la totalidad de los conflictos de intereses que hay en ella sin caer en contradicciones $^{4}$. De esta manera se han incorporado a la escena internacional nuevos sujetos distintos de los Estados, tales como organizaciones internacionales, comunidades supranacionales o regionales, empresas multinacionales y organizaciones no gubernamentales, las cuales desempeñan distintos roles que influyen decididamente en el ámbito jurídico internacional.

Las organizaciones internacionales como sujetos del derecho internacional contemporáneo, han adquirido protagonismo dentro de la comunidad mundial como una instancia

2 Escudero, Rafael. «El concepto de soft law». En: Moreso, José Juan y Martí, José Luis (eds.). Contribuciones a la Filosofia del Derecho. Editorial Marcial Pons, Madrid, 2012, p. 97.

3 LAGos, Enrique. «Algunas tendencias del Derecho Internacional a principios del S. XXI». Anuario Mexicano de Derecho Internacional, vol. V, 2005, p. 310

4 Escudero, loc. cit. 
para intentar resolver los problemas que afecten a dicha comunidad. Dentro de éstos, los foros multilaterales posibilitan la agrupación de los Estados en razón de sus intereses comunes, para negociar y alcanzar acuerdos, otorgando transparencia y permitiendo un mayor seguimiento del cumplimiento de los mismos 5 .

En este contexto, los organismos y conferencias internacionales son claves para la expansión del soft law, que en sentido amplio es:

"el derecho que generalmente se expresa a través de declaraciones y resoluciones y acuerdos ejecutivos, abarcando una vastedad de temas y, dentro de este contexto, haciendo posible un marco de referencia temporal, necesario en una sociedad en permanente cambio, frente a la ausencia de normas consolidadas en tratados o a través de la costumbre"6.

Sin embargo, esta noción no ha estado carente de discusiones y confusiones, pues si bien goza de gran preferencia por la libertad que otorga a los sujetos al brindarles la oportunidad de elección de principios, estándares y recomendaciones a las cuales someterse es también, por otro lado, una importante fuente de incertezas, las cuales a lo largo de este trabajo abordaremos e intentaremos despejar.

\section{Concepto de soft law}

Como señalamos en líneas anteriores, la aceleración del proceso de transformación del derecho internacional se debe, en gran medida, al fenómeno de la globalización y a la intervención e interacción de los diversos interesados. En razón a esto, el ordenamiento jurídico ha debido actualizarse para satisfacer las nuevas problemáticas internacionales, como las causas humanitarias, protección al medio ambiente, cuestión del cambio climático, revolución tecnológica, creación de instituciones supranacionales políticas o judiciales, entre otras ${ }^{7}$. La respuesta del ordenamiento jurídico ha implicado la necesidad de regular nuevas materias, antes no previstas, y adaptar el sistema de fuentes de creación de normas a los nuevos tiempos, a través de medidas tales como el denominado soft law.

Si bien el soft law ha alcanzado un mayor desarrollo en el último siglo, su origen no es nuevo, ya que sus primeros esbozos se remontan al siglo I a.C., cuando el derecho romano y sus exégetas ya estudiaban y teorizaban figuras normativas muy próximas a lo que hoy conocemos como soft law.

LaGos, op. cit., pp. 319-320.

Idem. p. 320.

Del Toro, Mauricio Iván. «El fenómeno de soft law y las nuevas perspectivas del Derecho Internacional». Anuario Mexicano de Derecho Internacional, vol. VI, 2006, p. 514.

8 Sarmiento, Daniel. El Soft Law Administrativo. Editorial Thomson Civitas, Navarra, 2008, p. 75. 
No obstante ello, la doctrina reconoce que el concepto de soft law fue acuñado por Lord $\mathrm{McNair}^{9-10}$, pero contando con diversas interpretaciones. Principalmente esta noción se ha atribuido para distinguir entre proposiciones lege ferenda y lege data; empero, no para distinguir las variantes normativas que van desde lo no vinculante a lo vinculante, de lo soft a lo hard. Siguiendo a Del Toro, creemos que con la expresión soft law se intenta describir enunciados formulados como principios abstractos y presentes en todo ordenamiento, y provenientes de su aplicación judicial ${ }^{11}$.

En 1983 Prosper Weil publicó "Towards relative normativity in international law", y según D`amato, Weil utilizaba el término soft law para caracterizar algunas normas que no son legalmente vinculantes, pero que a menudo son preferibles ante una falta de acuerdo $^{12}$. De ahí el temor de Weil en que el soft law pueda actuar como imán tirando hacia abajo las normas del derecho internacional ${ }^{13}$, y en consecuencia, las fuentes de derecho internacional pueden caer en desuso causando su deslegitimación.

Pese al transcurso de los ańos y de los intentos fallidos por conceptualizarlo, la doctrina no ha logrado arribar a un significado unívoco de soft law, pues por un lado se refiere a instrumentos heterogéneos en los que suelen concurrir dos elementos: el carácter no jurídicamente vinculante y una cierta relevancia jurídica, esto es, aquellos que provengan de resoluciones no vinculantes emanadas de organizaciones internacionales, instrumentos de actores no estatales y los denominados acuerdos no normativos. Y por otro lado, encontramos la expresión referida al contenido de instrumentos, ya sean jurídicamente vinculantes (legal soft law) o no (instrumentos doblemente soft $)^{14}$. Lo anterior se expresa en la definición de Snyder, quien señala que el soft law se compone de normas de conducta que, en principio, no tienen carácter jurídicamente vinculante, pero que sin embargo pueden tener efectos prácticos ${ }^{15}$.

En un sentido similar, Gersen y Posner entregan su propia definición de soft law, como "una regla emanada de una autoridad legisladora que no cumple con las formalidades

9 Arnold Duncan McNair fue el primer presidente del Tribunal Europeo de Derechos Humanos (19591965). Previamente, había sido presidente de la Corte Internacional de Justicia, durante los años 19521955.

10 Mazuelos, Ángeles. «Soft Law: ¿Mucho ruido y pocas nueces?». Revista electrónica de estudios internacionales, $\mathrm{n}^{\circ} 8$, 2004. p. 1 .

11 Del Toro, op. cit., p. 515.

12 D'Aмато, Anthony. " $A$ few steps towards an explanatory theory of International Law» [en línea]. Faculty Working Papers. Paper 90, 2010, p. 5. Disponible en: <http://scholarlycommons.law.northwestern.edu/cgi/ viewcontent.cgi?article=1089\&context=facultyworkingpapers $>$ [fecha de consulta: 18 de octubre de 2014]. Ibidem.

4 Mazuelos, op. cit., p. 2.

15 SNyder, Francis. «Soft law and institutional practice in the European Community». European University Institute Working Paper Law, n 93/5, 2003, p. 2. 
constitucionales y de otra índole que sean necesarias para que la norma sea jurídicamente vinculante. Definimos hard law como una regla emanada de una autoridad legislativa que sí cumple con las formalidades constitucionales y de otra índole que sean necesarias para que la norma sea jurídicamente vinculante", y agregan que "el ente legislador usa el soft law porque la aproximación del hard law tiene desventajas; a veces, pero no siempre, el soft law producirá los mismos efectos de comportamiento que una norma equivalente de hard law habría producido; en otras ocasiones, el soft law podría tener consecuencias más deseables que el equivalente más similar de hard law podría tener" ${ }^{16}$.

Por consiguiente, el soft law alberga instrumentos cuya juridicidad es dudosa o cuya fuerza vinculante es cuestionada al estar desprovistas de sanción ${ }^{17}$. Sin perjuicio de ello, no debe caerse en la confusión que todo aquello carente de obligatoriedad corresponde a soft law.

En este sentido, Guzmán y Meyer señalan que "el soft law se entiende de mejor manera como un continuo, o espectro, que se mueve entre tratados completamente vinculantes y posturas netamente políticas. Visto de esta manera, el soft law es algo que pierde su importancia a medida que los compromisos de los Estados se debilitan, eventualmente desapareciendo por completo." 18

Así las cosas, la clave del soft law radicaría en la descripción de los fenómenos que comprende y la sujeción al concepto de Derecho que se adopte, ya que generalmente el término law evoca una obligación jurídica, generando para algunos reticencias al verlo empleado en relación con fenómenos donde ésta no se verifica ${ }^{19}$.

Sin perjuicio de lo anterior, no hay que perder de vista que "el orden jurídico internacional ya no es un universo cerrado, y hoy los miembros de la comunidad internacional juegan un rol activo en la generación de soluciones, pudiendo acordar nuevas fórmulas para crear derechos, cubriendo lagunas en las cuales la legislación es insuficiente, ineficaz o carece de consenso ${ }^{20}$, o satisfaciendo nuevos intereses de la comunidad internacional, que de otro modo se tornaría complejo, al mismo tiempo de ir enriqueciendo aún más el soft law, dotándolo de nuevas características para dar respuesta a nuevos problemas.

16 Gersen, Jacob y Posner, Eric. "Soft Law». Stanford Law Review, vol. 63, n 3, p. 579.

17 Mazuelos, op. cit., p. 2.

18 Guzman, Andrew y Meyer, Timothy. «International Soft Law». The Journal of Legal Analysis, vol. 2, $\mathrm{n}^{\circ} 1$, 2011, p. 173.

19 Ididem.

20 Boretto, Mónica. «Soft Law. Nuevos enfoques para el desarrollo progresivo del Derecho Internacional de la Propiedad Intelectual». Ars Boni et Aequi, vol. 8, n 2, 2012, p. 85. 


\section{Naturaleza jurídica}

Gracias a la transformación de los Estados, por influjo de los procesos de integración económica, se han modificado las formas de creación de Derecho, y el soft law es una evidencia de la adaptación a este nuevo contexto global, puesto que la realidad reclama efectividad inmediata y activismo en su adaptabilidad al nuevo entorno.

En este nuevo escenario emerge el paradigma liberal de la autonomía de la voluntad, permitiendo la adaptabilidad a los cambios, ya que son las mismas partes y las realidades quienes van transformando el derecho ${ }^{21}$. De este modo, el soft law se constituye como una fuente alternativa, caracterizada por su falta de coercitividad, desapego a la rigidez del principio de legalidad e indeterminada en su contenido, alcanzando todos los actos e instrumentos jurídicos sin carácter obligatorio en sentido formal ${ }^{22}$.

Esta anómala naturaleza del soft law es analizada por Boretto desde diversas perspectivas. Así, desde una perspectiva analítica, el soft law posee una naturaleza híbrida, constituida por reglas y principios. Estos últimos -los principios- son mandatos de optimización, de contenido determinado en su antecedente, pero indeterminado en sus consecuencias. En efecto, el contenido abierto e indeterminado de las consecuencias jurídicas del principio, es el que le atribuye a la norma de soft law un carácter más vinculado a la optimización o maximización de resultados, que al cumplimiento taxativo de los mismos, pues se caracterizan por el hecho de que pueden ser cumplidos en diferentes grados, y la medida debida de su cumplimiento no sólo depende de las posibilidades reales, sino también de las jurídicas (de las dispuestas en el propio ordenamiento). En cambio, las reglas son normas que sólo pueden ser cumplidas o $n^{23}$ y es la que no existe gradualidad en su cumplimiento.

En otro sentido, desde una perspectiva ontológica, el soft law se caracteriza por contar con presupuestos taxativos de consecuentes abiertos e indeterminados, que reclaman la maximización de sus objetivos ${ }^{24}$, es decir, se trata de un mandato de optimización, pero tendrá efectos jurídicos dependiendo del sistema formal de fuentes, pues de existir una norma de reenvío que le reconozca su fuerza vinculante, ya no sería posible continuar hablando de soft law, sino que se trataría de hard law.

Desde la esfera empírica, cada acto o instrumento de soft law puede tener una estructura

Del Toro, op. cit., p. 533.

BoretTo, op. cit., p. 85.

Ibidem.

Ídem, p. 86. 
de regla o de principio. En la praxis existen instrumentos que se articulan como reglas, estableciendo un antecedente y un consecuente determinado, los cuales implican un mayor grado de compromiso de sus destinatarios, y otros simplemente con un consecuente indeterminado. Por tanto, desde esta perspectiva, el soft law comprende reglas o principios $^{25}$.

Por su parte, desde un plano formal, Boretto señala que no puede ser considerado el soft law dentro de la jerarquía de las fuentes enunciadas en el artículo 38 del Estatuto de la Corte Internacional de Justicia ${ }^{26}$, puesto que dicha norma muestra una concepción estática bastante alejada de la realidad, que excluye a las resoluciones adoptadas con el rango de compromiso entre las partes. En este sentido, compartimos con Boretto en sostener como instrumentos excluidos del mencionado artículo a las resoluciones no obligatorias de los organismos internacionales, resoluciones de actores no estatales, acuerdos interestatales no normativos y soft law material ${ }^{27}$.

No obstante lo anterior, el mismo artículo reconoce la facultad de la Corte para decidir un litigio ex aequo et bono si las partes así lo convinieren, en otras palabras, para resolverlo en razón de principios de equidad y justicia. Por tanto, es menester indicar que si bien el soft law no ha sido reconocido abiertamente por las legislaciones producto de la naturaleza anómala de éste, se han establecido vías que dan paso a la discrecionalidad y ejercicio de autonomía de la voluntad de las partes.

\section{Características}

Tal como esbozamos anteriormente, uno de los rasgos esenciales del régimen del soft law, en contraposición al hard law, es la participación y aporte de recursos de actores no gubernamentales en la construcción, operación e implementación de instrumentos del soft law.

\section{Ibidem.}

26 El Estatuto de la Corte Internacional de Justicia, en su Art 38 señala: “1. La Corte, cuya función es decidir conforme al derecho internacional las controversias que le sean sometidas, deberá aplicar: a. las convenciones internacionales, sean generales o particulares, que establecen reglas expresamente reconocidas por los Estados litigantes; b. la costumbre internacional como prueba de una práctica generalmente aceptada como derecho; c. los principios generales de derecho reconocidos por las naciones civilizadas; $d$. las decisiones judiciales y las doctrinas de los publicistas de mayor competencia de las distintas naciones, como medio auxiliar para la determinación de las reglas de derecho, sin perjuicio de lo dispuesto en el Artículo 59.

2. La presente disposición no restringe la facultad de la Corte para decidir un litigio ex aequo et bono, si las partes así lo convinieren".

27 Boretto, loc. cit. 
Suma a lo anterior que el soft law goza de mayor claridad en su configuración en relación al hard law. De este modo, dentro de sus características podemos señalar, en primer lugar, que en un régimen de soft law no se depende de la autoridad legal, regulatoria y formal de un gobierno, o inclusive puede ni siquiera incluirse en el diseño y operación institucional. En segundo lugar, los operadores actúan de manera voluntaria en la construcción, operación y mantenimiento de acuerdos, pudiendo cualquier participante retirarse en cualquier momento, o adherir al régimen, sin necesidad de invocar potestad sancionadora de la autoridad estatal. En tercer lugar, el soft law posee una fuerte dependencia en el consenso de adopción de decisiones para generar acciones, y más ampliamente como una fuente de unión institucional y de legitimidad. Y en cuarto lugar, y que fluye de la tercera característica, el poder estatal-policial como forma de inducir el consentimiento y el cumplimiento está ausente ${ }^{28}$.

Por su parte, Boretto ${ }^{29}$ distingue otros elementos característicos del soft law, tales como: a) el carácter jurídicamente no vinculante, b) cierta relevancia jurídica, c) buena fe, elemento presente y dominante en las relaciones interestatales, así como en los acuerdos que derivan de las mismas, y d) no aplicabilidad de sanciones como consecuencia de su incumplimiento.

En relación a las dos últimas características, consideramos pertinente sostener que el soft law tiene un alcance relativo, pues aun cuando el incumplimiento de sus disposiciones no fuesen sancionables mediante las formas tradicionales, existe el soft law coercitivo, que fluye desde la perspectiva social y no jurídica propiamente tal, a través del cual la sociedad civil en un escenario de democracia participativa interviene en los procesos de decisión de asuntos públicos.

En suma, y dadas las características del soft law, se trata de una herramienta que cada día alcanza mayor relevancia y participación en el ordenamiento jurídico internacional, y que puede adquirir diferentes formas, incluyendo aquellas en las cuales las resoluciones del gobierno están presentes pero no lo dominan. Un ejemplo de lo anterior lo constituyen las organizaciones intergubernamentales, como la Organización para la Cooperación y Desarrollo Económico (OCDE), las cuales emplean el soft law al contar con la participación tripartita de gobiernos, empresas y trabajadores para diseñar y administrar los códigos que ellos voluntariamente producen para sus miembros, y contando con diversos niveles de libertad para aceptarlos o no. De manera más amplia aún, el soft law puede albergar al derecho consuetudinario internacional y a prácticas estatales, codificadas o no, incorporando de este modo el derecho internacional público

28 Kirton, John y Trebilcock, Michael (eds.). Hard Choices, Soft Law. Ashgate, 2004, p. 9.

29 Boretto, op. cit., p. 84. 
a los numerosos sistemas que surgen, como el arbitraje internacional comercial -bajo el régimen de derecho internacional privado-, lo cual no implica necesariamente que el soft law se constituya de costumbre internacional. En efecto, usualmente se trata de una mezcla de la autoridad pública en forma de instituciones intergubernamentales con más autoridad privada, inspiradas en prácticas como las del Tratado de Libre Comercio de América del Norte (NAFTA), las cuales dependen de sistemas basados en arbitraje comercial privado, como se plasma en el Centro Internacional de Arreglo de Diferencias Relativas a Inversiones (CIADI) del Banco Mundial ${ }^{30}$.

Causa de ello es que el cumplimiento del soft law no sólo está determinado por el cumplimiento de normas no vinculantes, sino además por la observancia de las conductas de los sujetos por parte de la comunidad internacional, que mediante la presión social, política y económica instan al cumplimiento y comportamiento de acuerdo a estos estándares.

\section{Expresiones de soft law}

La eficacia de las herramientas soft law dependerá de la participación activa de los ciudadanos, de la publicidad adversa, presiones sociales, apreciación pública y reacciones voluntarias del mercado de consumidores, empleados e inversores. Es menester señalar que usualmente estos instrumentos no contienen formas legales o sanciones económicas.

Manifestación de lo anterior son las recomendaciones de la Organización Internacional del Trabajo (OIT), las cuales complementan los convenios de la misma OIT y contienen directrices para la política nacional en materia laboral, fijando de este modo estándares internacionales que tienen forma de soft law, pues carecen de fuerza obligatoria, por lo que para generar efectos jurídicos se requiere de la acogida de éstas por parte de los Estados; y a su vez, la OIT le otorga asistencia técnica para implementarlas. Sin embargo, constantemente se critica la ineficacia del sistema de normas internacionales del trabajo de la OIT, pues sólo los convenios y protocolos gozan de fuerza vinculante, mas no las recomendaciones.

Semejante es lo que ocurre con otra clase de instrumentos de soft law consistente en una serie de mecanismos voluntarios de certificación, etiquetado y códigos de conducta, con el objetivo de identificar empresas o productos que se ajusten a las normas internacionales del trabajo, y por lo tanto responder a las fallas de información de mercado cuando se producen violaciones a los derechos de los trabajadores ${ }^{31}$.

30 Kirton yTrebilcock, op. cit., p. 9.

31 Idem, p. 175. 
La eficacia de éstos resulta en gran medida de las reacciones del mercado a las señales de los consumidores y, en menor medida, de los inversionistas. Estos instrumentos son atractivos en algunos aspectos desde el enfoque del bienestar del consumidor, que dependerán de las preferencias de consumo. Normalmente estos mecanismos son iniciados por las propias sociedades industriales o por ONG's de diversos tipos, las cuales negocian entre ellas o con otras sociedades comerciales los instrumentos de soft law tendientes a promover el cumplimiento de estándares internacionales laborales.

El contenido normativo de las resoluciones de soft law, en principio, no goza de un valor obligatorio, pese a contener el sustrato ético del derecho internacional, configurándose a través de principios y objetivos ${ }^{32}$. Empero, esto generaba poca claridad entre lege data y lege ferenda, al no poder distinguir entre la norma existente y la norma futura. Con posterioridad, surgió la interrogante acerca de la posibilidad que una norma no vinculante perteneciente al soft law fuera capaz de presionar en su cumplimiento en virtud de la suscripción de tratados internacionales.

Así, los efectos jurídicos de estas disposiciones no vinculantes constituyen la principal controversia, puesto que si las previsiones de los tratados constitutivos implicasen obligaciones para los Estados miembros, éstas no se podrían generar a partir de las recomendaciones per se, sino de su conjunción con alguna fuente de obligaciones jurídicas ${ }^{33}$.

En tanto, las recomendaciones son instrumentos a través de los cuales se incita al logro de los objetivos de una organización internacional, por parte de los órganos facultados para ello ${ }^{34}$. En estas organizaciones internacionales, el principio de buena fe juega un rol trascendental, ya que refleja el principio de cooperación internacional, inherente a las mismas, constituyendo de este modo fuente de obligaciones jurídicas para los estados miembros, que de buena fe deben observar lo recomendado ${ }^{35}$.

Gloria Alarcón, citando a Camaño Anido y Calderón Carrero, señala que determinadas organizaciones internacionales, como la OCDE y la OMC, o bien otras instituciones internacionales, como el Banco Mundial o el Fondo Monetario Internacional, quienes elaboran reglas, directrices, lineamientos, catálogos, códigos de conducta, etc., lo realizan con el objeto de establecer o codificar una serie de parámetros uniformes a nivel internacional, con la finalidad de que tanto los países miembros como los no miembros de dichas organizaciones, admitan lo que en la mayoría de los supuestos les conducirá a reformar su legislación interna a tal efecto. Un ejemplo paradigmático de este tipo de soft law-o de "backdoor rules" en la terminología de los autores citados- lo constituyen

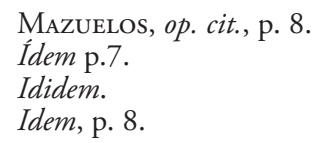


los Modelos de Convenio para evitar la doble imposición recomendada por el Comité Fiscal OCDE y sus comentarios, o las Directrices sobre Precios de Transferencia o sobre competencia fiscal perniciosa elaboradas por este mismo comité, las cuarenta Recomendaciones en materia antiblanqueo del Financial Action Task Force, o las Directrices que utiliza el FMI o el Banco Mundial cuando negocian con los diferentes gobiernos estatales el otorgamiento de créditos internacionales ${ }^{36}$.

Como señalábamos en párrafos anteriores, las recomendaciones de la AGNU tienen un carácter propositivo, invitando a adoptar determinadas conductas. Sin embargo, existen otras resoluciones de las AGNU que:

“...por su contenido, circunstancias de su adopción y la práctica de los Estados, son especialmente relevantes en el proceso de elaboración del derecho internacional. Se trata de resoluciones cuyo contenido se refiere a principios o normas jurídicas. No es extraño, por tanto, que sean citadas repetidamente, evidenciando convicciones arraigadas en los Estados. Se adoptaban por unanimidad, consenso o mayorías representativas de la comunidad internacional. Suelen ser el resultado de largas negociaciones, su redacción es cuidada y se adoptan con menos frecuencia que las meras recomendaciones..." ${ }^{37}$.

Según Bell y McGillivray ${ }^{38}$, el documento de soft law más importante es la Agenda 21. En palabras de los autores:

"In international law, sustainable development as a legal concept has tended to be found mostly in "soft law" - that is, in documents that are not directly binding and which have more of a policy feel to them. The most important document here is Agenda 21, signed at the 1992 Rio Earth Summit, which is essentially a lengthy blueprint for realizing sustainable development. This pays particular attention to action at the local level and picks up many of the recommendations made in the Brundtland Report ${ }^{39}$ about greater public involvement in decision making through, for example, access to environmental information. There are signs that as least some of the judges of the international Court of Justice (ICJ) are beginning

36 Alarcón, Gloria. El Soft Law y nuestro sistema de fuentes [en línea], 2010, p. 16. Disponible en: <http:// digitum.um.es/xmlui/bitstream/10201/10423/1/El\%20soft\%20law\%20y\%20nuestro\%20Sistema\%20de\%20 fuente\%20\%20Homenaje\%20RodriguezBereijo\%20\%20pre-print\%2017\%2002\%202010.pdfs [Fecha de consulta: 20 de octubre de 2014].

37 Mazuelos, op. cit., p. 8.

38 Bell, Stuart y Mcgillivray, Donald. Environmental Law, Seventh edition. Oxford University Press, New York, 2008, p. 57.

39 El Informe Brundtland fue elaborado en 1987 por diversos países para Naciones Unidas, con el objetivo de aportar una definición de desarrollo sostenible, examinar temas críticos de desarrollo y medio ambiente y formular propuestas y soluciones al respecto. 
to recognize the procedural dimension tissues of sustainable development and to recognize sustainable development as a principle rather than only as a concept" ${ }^{\text {"40. }}$.

Sin perjuicio del reconocimiento que puede llegar a otorgársele al interior de la Corte Internacional de Justicia, y más allá de los acuerdos sobre requisitos básicos de procedimientos sobre la participación pública mencionados en la Declaración de Río, no existe un consenso jurídico internacional sobre el desarrollo sostenible. Asimismo, no se habría progresado lo suficiente en la materia en la Cumbre Mundial sobre el Desarrollo Sostenible, celebrada en Johannesburgo el año 2002. Ello se debería, entre otras razones, a que las diversas convenciones o declaraciones de principios adoptadas en la Conferencia de Río, entre ellas la Declaración sobre el Desarrollo Sostenible, contenían múltiples aspiraciones pero carecían de carácter vinculante ${ }^{41}$.

En materia comercial, como veremos posteriormente, los acuerdos internacionales asumen contenidos abiertos e indeterminados que requieren de la colaboración normativa de los instrumentos de soft law, tal como sucede con los acuerdos alcanzados al amparo de la Organización Mundial de Comercio o con las decisiones y declaraciones que integran el Acervo de la Ronda de Uruguay, y que tienen por objeto darle sentido a los diversos acuerdos multilaterales.

En la misma vereda, cabe mencionar el caso de las cuarenta recomendaciones adoptadas por el Grupo de Acción Financiera sobre el Blanqueo de Capitales (GAFI), mediante las cuales se busca evitar el tráfico ilícito de capital, al cual se suman sus notas interpretativas ${ }^{42}$. El carácter atípico de las cuarenta recomendaciones no fue impedimento para que los Estados introdujeran su contenido en normas internas de hard law. Ejemplo de ello es la adopción de la Directiva 91/308/CEE del Consejo, en la Comunidad Europea, relativa a la prevención de la utilización del sistema financiero para el blanqueo de capitales, introduciendo en los veintisiete Estados miembros las cuarenta recomendaciones ${ }^{43}$.

De este modo, los foros de diversas convenciones y las organizaciones internacionales llegan a participar en el proceso de creación normativa ${ }^{44}$.

¿Es suficiente que una norma sea no vinculante para que sea considerada parte del soft law?

Como sostiene Mazuelos, el concepto de soft law constituye una noción imprecisa, pues alberga diferentes situaciones e incluso algunas difíciles de prever, ya que muchos de los

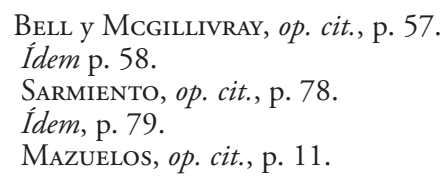


acuerdos internacionales tienen estándares vagos, lo que hace necesaria la intervención de órganos internacionales. Si bien se suele asociar la falta de precisión con el soft law, en realidad su característica distintiva es la no exigibilidad de la obligación. Esto es, su no obligatoriedad, lo que confiriere a las partes de un amplio margen de discrecionalidad en el cumplimiento de las obligaciones contraídas ${ }^{45}$.

\section{Soft law y su relación con el derecho comercial}

El comercio internacional y la creación de políticas económicas a escala mundial son fenómenos ligados al soft law. Las dificultades inherentes a la puesta en práctica del derecho económico internacional justifican la adopción de actos e instrumentos flexibles, ajenos a la rigidez del derecho internacional convencional. Pese a gozar de una larga trayectoria y de un alto grado de institucionalización (FMI, Banco Mundial, OMC, OCDE, etc.), lo cierto es que en el derecho económico internacional poco a poco el soft law ha ido adquiriendo mayor importancia ${ }^{46}$.

Así, la proliferación de leyes modelo elaboradas en foros internacionales, algunos actos normativos característicos del derecho institucional, convenios internacionales disponibles por las partes o la propia fuerza ejemplificadora de determinados convenios internacionales, actúan a menudo como fuentes de derecho en sentido impropio ${ }^{47}$. No es sorprendente que se haya recurrido cada vez más a las soluciones de soft law ante las complejidades del actual orden mundial económico, ya que estas poseen una singular flexibilidad. Existen dos tipos principales de instrumentos para su aplicación. Unos son los estándares voluntarios, que sirven como equivalente a las leyes formales. Los otros surgen de instituciones informales en los planos internacional, transnacional y nacional, con una fuerte dependencia de la participación voluntaria, los recursos y las acciones consensuadas por sus miembros. Estos evalúan regularmente las contribuciones obligatorias, organización, recursos y las sanciones de la propia institución. Ejemplos de esto son, en el sector químico, el Programa de Cuidado Responsable (Responsible Care Programme), o en términos más amplios la Organización Internacional de Normalización (ISO) ${ }^{48}$.

Durante la última década se ha vivido una intensa explosión de estas normas voluntarias, habiéndose aplicado en la mayoría y más importantes sectores industriales de la economía y el intercambio a nivel global ${ }^{49}$.

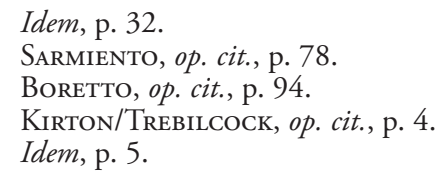


Tal vez la principal causa de lo anterior radique en la capacidad del soft law de avanzar hacia la unificación del derecho sin soportar los costos de negociación que supone un proceso formal.

Sin embargo, un abuso del soft law podría responder, según los casos, a una búsqueda de fuga del derecho que pretende proponer un nuevo realismo jurídico, tendiente a identificar el fundamento del derecho con la doctrina del derecho ${ }^{50}$.

En otro sentido, el derecho del comercio internacional es una realidad que sólo en ciertas ocasiones ha encontrado respuestas por la vía del derecho uniforme, dado los límites y dificultades que éste impone. Muchos sectores claves del comercio internacional siguen presentando una pluralidad de técnicas normativas, sin prescindir de las conflictuales, incluso en aquellos casos en que se ha logrado una cierta uniformidad ${ }^{51}$. Esto se debe a que, en el dinámico contexto del mercado internacional, opciones como la del soft law resultan especialmente apropiadas por la eficiencia de sus respuestas y soluciones, por su inmediatez y por adecuarse a los intereses de las partes contratantes.

También trabajan con regulaciones de soft law iniciativas tales como el Programa de Naciones Unidas para el Medio Ambiente, PNUMA, que tiene por finalidad promover acuerdos en materia medioambiental a través de acuerdos vinculantes, declaraciones o acuerdos de intención, entre otros. Del mismo modo, la Organización de Cooperación y el Desarrollo Económico (OCDE) otorga un marco a los Estados para buscar soluciones a problemas comunes, identificando buenas prácticas y colaborando en la elaboración de políticas internas en razón de estándares internacionales.

Otro caso emblemático de soft law son las guías o normas técnicas emitidas por entidades privadas respecto a las características que debe reunir un determinado producto, bien o servicio. Los Best Available Techniques Reference Document (BREFs) son guías en las que se recogen las mejores técnicas disponibles en una determinada materia, y sirven para evaluar y controlar la actividad relativa a la misma. Suelen elaborarse mediante grupos de trabajo, con participación de expertos, representantes de diversas administraciones y sectores afectados, buscando la legitimidad del proceso. Junto a ello pueden introducirse normas con el carácter de cláusulas técnicas, cuyo cumplimiento o no podría llevar a entregar una concesión o a revocar una licencia o permiso ${ }^{52}$.

BoretTo, op. cit., p. 95.

Idem, p. 93.

Idem, p. 110. 


\section{Conclusiones}

Resulta indiscutible que el concepto de soft law acarrea una serie de confusiones. Sin embargo, pareciera que existe al menos un consenso respecto a que se trataría de una categoría descriptiva que implica la falta de obligatoriedad jurídica de los instrumentos internacionales.

Existen expresiones que reflejan de mejor manera el carácter del soft law, tales como las "recomendaciones", puesto que devienen de la voluntad de los sujetos de derecho internacional, y en las cuales previamente han pactado principios y estándares de conducta, que posteriormente son invitados a seguir.

Es necesario considerar que la fortaleza del soft law, su naturaleza no vinculante, puede sin embargo convertirse en su principal debilidad cuando los contextos en los que se aplica no son los adecuados para lograr consenso social.

El soft law enfrenta un proceso de fortalecimiento gracias al éxito de sus instrumentos, que permiten el diseńo de variadas opciones conforme las necesidades de las partes. 
